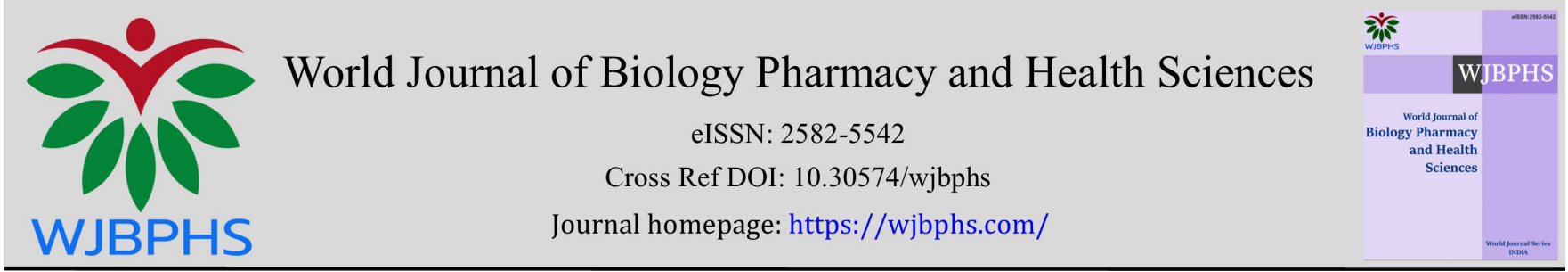

(RESEARCH ARTiCLE)

Check for updates

\title{
Anti-plasmodial Effect of C. limon and C. paradisi extracts on Plasmodium berghei- infected mice
}

Michael Okpara Elom 1, ${ }^{*}$, Anthony Gideon Uche 2, Boniface Nwofoke Ukwah 1, Victor Udoh Usanga 1, Anthonia Ifeoma Okpara-Elom ${ }^{2}$, Michael Erem Kalu ${ }^{1}$ and Onyekachi Ewa Ibe ${ }^{1}$

\author{
${ }^{1}$ Department of Medical Laboratory Science, Ebonyi State University, Abakaliki, Nigeria. \\ 2 Department of Applied Microbiology, Ebonyi State University, Abakaliki, Nigeria.
}

World Journal of Biology Pharmacy and Health Sciences, 2021, 08(02), 026-033

Publication history: Received on 02 October 2021; revised on 10 November 2021; accepted on 12 November 2021

Article DOI: https://doi.org/10.30574/wjbphs.2021.8.2.0118

\begin{abstract}
Antiplasmodial effect of Citrus limon and Citrus paradisi extracts on Plasmodium berghei-infected mice was studied. Twenty five albino mice were randomized into five categories of G, L, GL, ACT (positive control) and NC which stand for grape, lemon, grape and lemon combined extracts, artemisinin combined therapy and negative control respectively. The NC group did not receive any intervention. Other treatments were administered orally for 12 days whereas administration of ACT lasted for 3 days. Blood was collected from the tail vein of the mice at a three day interval through venipuncture. Thick blood films were prepared and parasite densities were estimated using standard parasitological techniques. Results were analysed with ANOVA and Duncan multiple range tests. There was no significant difference ( $p>0.05$ ) between parasite densities of the treatment groups and the negative control at baseline levels. However, as the treatment progressed from day 3 through day 9, there were significant reductions $(\mathrm{p}<0.05)$ in parasite densities among treatment groups when compared to the negative control. In this study, extracts of $C$. limon and $C$. paradisi in both single and combined strengths have been found to have antiplasmodial properties in mice. ACT possessed the highest antiplasmodial effect while C. limon as a single treatment ranked second in possession of antiplasmodial activity but exhibited increased RBC lysis. In combination, C. limoni and C. paradise extracts showed antiplasmodial activity that is slightly less than that exhibited by the lemon extract alone, but maintained normal RBC morphology whereas $C$. paradisi extract alone exhibited the lowest level of parasite clearance with atrophied red blood cells. Investigation of the effects of the extracts on liver, kidney and gastrointestinal tissues of mice is recommended before they could be prescribed as antimalaria for other animals and humans.
\end{abstract}

Keywords: Citrus limon; Citrus paradisi; Extracts; Plasmodium; Mice

\section{Introduction}

Malaria is an arthropod- borne parasitic infection that is transmissible in sub-Sahara Africa and other tropical and subtropical countries [1] where the prevailing environmental conditions support the survival and development of both the anopheline mosquito vectors and the parasites. The disease is transmitted by female anopheline mosquitoes that harbour the protozoan parasite that belongs to the genus known as Plasmodium. Both humans and animals suffer from malaria infections which are caused by different species of Plasmodium. P. falciparum, $P$. vivax, $P$. ovale, $P$. malariae and the recently discovered $P$. Knowlesi are responsible for human malaria whereas many other Plasmodium species cause malaria in rodents and other mammals. Symptoms of uncomplicated human malaria infection include fever, headache, chills, fatigue, malaise, shivering or sweating, pain in the muscles or abdomen and lower back, nausea, vomiting, and pallor $[2,3]$. It has been reported that the first symptoms might be mild and could be difficult to be recognized as malaria

\footnotetext{
${ }^{*}$ Corresponding author: MichaelOkpara Elom

Department of Medical Laboratory Science, Ebonyi State University, Abakaliki, Nigeria.

Copyright $(2021$ Author(s) retain the copyright of this article. This article is published under the terms of the Creative Commons Attribution Liscense 4.0.
} 
if left untreated within 24 hours and that under such a condition, P. falciparum malaria may advance to severe illness with fatal outcome [3]. Malaria- associated complications have been reported to include respiratory distress, encephalopathy, splenomegaly, hypoglycaemia, hepatomegaly, haemoglobinuria and coagulopathy $[4,5,6]$. P. falciparum is the most dangerous among the species and it can cause malaria with many associated complications and it is the most incriminated species in global malaria-related fatalities [7,8]. According to the World Health Organization, approximately $40 \%$ of the entire world population live in malaria endemic areas, where an estimated 300-500 million clinical cases occur, with approximately 1.5-2.7 million mortality cases recorded annually [9]. As at 2018, the WHO reported an estimate of 3.2 billion people as being at risk of malaria infection in 91 countries of the world [10]. Malaria is a life threatening disease with more devastating effect among young children and pregnant women [11]. Malaria in pregnancy can result in still birth, infant mortality, abortion and low birth weights of babies [12]. Pregnancy- associated maternal malaria can degenerate to life-threatening anaemia if not urgently and properly diagnosed and treated. Four Plasmodium species that infect the African thicket rats (Grammomys species and Thamnomys species) have been reportedly described and classified as P. berghei, P. yoelii, P. chabaudi and P.vinkei [13].

A common origin of rodent malaria parasites and their P. falciparum relatives has been reported [14]. The genome of $P$. berghei has been reportedly sequenced with resultant high similarity in both structure and content, with that of the human P. falciparum parasite; and some clinical malaria features arising from in vivo malaria studies in animal models such as mice have been reported to be similar to observations made in human studies [3].Such observations could be usefully extrapolated in the management and treatment of human malaria.

The emergence and rapid spread of multi-drug resistant strains of $P$. falciparum has become an important global issue of concern in clinical malaria chemotherapy [15] and this problem has been complicated by the development of multiple insecticide resistance by the mosquito vectors, thereby increasing the burden of malaria as a public health problem on the affected populations. This scenario has therefore stimulated the search for alternative treatment measures against the disease. The use of medicinal plant extracts against malaria is an age-old practice that has been considered a primitive treatment measure by poor populations. Nowadays, there is a contemporary change in perception that has created a paradigm shift in the utilization of orthodox malaria treatment options towards phytomedicine.

Citrus species such as grapefruits (C. paradisi) and lemon (C. limon) possess anti-oxidative, anti-bacterial, anti-diabetic and probably other hidden medicinal and nutritional beneficial properties. The medicinal properties of citrus have brought them into limelight as good candidates for experimental studies involving phytotherapeutic assessments. Forty-nine plants including Citrus species have been reported to possess anti-malarial properties [16].Phytochemicals are synthesised through plant metabolic processes and they have been reported to possess medicinal properties [17]. Crude extracts of alkaloid contents of lemon have been reported to possess both anticancer and antibacterial properties. Citrus flavonoids possess a large spectrum of biological activities and some anti pathogenic potential including antibacterial, anticancer, antifungal, antidiabetic, and antiviral activities [18]. Naringin, a predominant flavone in grapefruit, can remove free radicals and possess metal chelating and anti-oxidant properties [19,20]. Based on the antioxidant properties of vitamin $\mathrm{C}$ and oxidative stress involved in malaria infections, some of the malaria medicines are often prescribed with vitamin $\mathrm{C}$ or similar antioxidant supplements. Currently, malaria prevention and treatment seem eluding as the mosquito vectors have developed resistance to many available insecticides and some strains of Plasmodium species are rapidly acquiring resistance to the available orthodox malaria drugs. Therefore, there is a need for search for alternative treatments especially from the poorly exploited, harnessed and recognized traditional plants, hence, the need for this present study.

\section{Materials and methods}

\subsection{Acquisition of Plant Materials}

Fruits belonging to the same genus Citrus but of different species, C. limon and C. paradisi were bought from fruit vendors in Abakaliki, Ebonyi State, Nigeria.

\subsection{Preparation of Plant Materials for Extraction}

The fruits were separately cut in quadrants in order to expose the juice sacs and remove the seeds. The exocarps and endocarps were removed and only the juice sacs were collected. The collected juice sacs were put in an electric blender and blended for forty-five minutes so as to extract the juice from the juice sacs. The mixture was sieved to remove the empty juice sacs and extract the juices only. Twenty-five milliliters of $C$. limon and $C$. paradisi extracts were combined and homogenized. 


\subsection{Determination of Dry Mass of Extracts}

The extracts of both $C$. limon and $C$. paradisi were dried at $60^{\circ} \mathrm{C}$ in a hot air oven in order to obtain the dry mass of the extracts. After drying, the extracts were collected, weighed and stored in readiness for use.

\subsection{Experimental Animals}

The experimental animals were healthy one month old male mice that were bred and housed under standard animal house conditions in the Faculty of Veterinary Medicine of the University of Nigeria, Nsukka, Enugu State. The mice were six (6) in a group and made up of three (3) replicates. The range of the weights of the mice was $100 \mathrm{~g}-200 \mathrm{~g}$.

\subsection{Parasite Inoculation}

The rodent malaria parasite, $P$. berghei ANKA strain that is resistant to chloroquine was obtained from Parasitology Unit of Faculty of Veterinary Medicine, University of Nigeria Nsukka, Enugu State. The healthy mice were inoculated by injecting $0.2 \mathrm{ml}$ of $P$. berghei-infected blood specimen. The blood was diluted with normal saline in $1: 10^{8}$ to contain $2 \times$ $10^{7}$ parasitized red blood cells as described by Carter and Diggs [21].

\subsection{Baseline Analysis}

Venous blood from the tail of the mice were collected and examined for confirmation of parasitaemia; using thick film stained with Giemsa. The parasite- infected cells were counted according to Cheesbrough [22].

Parasite density per microliter of blood was calculated using assumed white blood cell count of 8000 as described by Haggaz et al. [23]. It was determined by calculating

$$
\frac{\text { Number of Parasites counted }}{200 \text { White Blood Cells(WBC) counted }} \times 8000 \mathrm{WBC} / \mu L
$$

\subsection{Administration of the Extracts}

After being infected, the mice were orally-fed with the freshly- extracted fruit juice according to their groups. Two (2) microliter per gram body weight of mice of the fresh juice extracts was administered once daily for 12 days. Thereafter, the animals were tested for malaria parasites. The extracts were administered as stated below:

Group 1 - received C. paradisi extract only.

Group 2 - received $C$. limon extract only.

Group 3 - received a combination of both $C$. paradisi and $C$. limon extracts.

Group 4 - received ACT solution as standard antimalarial drug (Positive Control) and

Group 5- untreated group (Negative Control)

\subsection{Determination of Antiplasmodial Activity of the Extracts}

Post-treatment blood specimen was collected from mice of each treatment group through tail venipuncture and thick film was made as described by Cheesbrough [22]. The thick blood film was air-dried and stained with Giemsa. The stained film was examined with x100 objective lens, using immersion oil. Parasite densities were estimated using assumed WBC count of 8000 and expressed per microlitre of blood.

\subsection{Data Analysis}

Data were analyzed using SPSS version 20.0 software and were subjected to analysis of variance (ANOVA). Means were separated using Duncan multiple range tests (DMRTs). Levels of significance were established at $\mathrm{p}<0.05$.

\section{Results}

Table 1 indicated no significant difference $(\mathrm{p}>0.05)$ in mean parasite densities between the treatment groups that received grape extracts $(G=216.00 \pm 9.80)$, Lemon $(\mathrm{L}=216.00 \pm 17.89)$ and grape and lemon in combination $(\mathrm{GL}=$ $216.00 \pm 9.79)$ and the group that received standard drug (ACT $=248.00 \pm 14.97)$ when compared to the negative control that was not treated with any form of drug or extract $(\mathrm{PC}=216.00 \pm 9.80) .0 n$ day 3 of the treatment period, the levels of parasitaemia of the groups that received grape extract $(G=152.00 \pm 8.00)$, Lemon $(L=192.00 \pm 14.97)$, grape and lemon combined $(\mathrm{GL}=160.00 \pm 12.65)$ and standard drug (ACT = $160.00 \pm 17.89$ ), dropped significantly when compared to the negative control group $(\mathrm{NC}=312.00 \pm 14.97)$. There was marked significant difference $(\mathrm{p}<0.05)$ 
between the groups that received treatments (extracts and standard drug) when compared to the negative control group (Table 2). On the 6th day of treatment as depicted in figure 1, the parasite density levels in the groups that received extracts and standard drug, significantly $(\mathrm{p}<0.05)$ reduced $(\mathrm{G}=104.00 \pm 9.80, \mathrm{~L}=152.00 \pm 14.97, \mathrm{GL}=104.00$ $\pm 9.80)$ and standard drug $(\mathrm{ACT}=120.00 \pm 17.89)$ when compared to the negative control $(\mathrm{NC}=344.00 \pm 16.00) .0 \mathrm{n}$ the 9th day of treatment as depicted in figure 2, the parasite density levels of groups that received extracts $(\mathrm{G}=88.00 \pm$ $8.00 ; \mathrm{L}=104.00 \pm 9.8, \mathrm{GL}=80.00 \pm 12.65)$ and those of the group that received standard $\operatorname{drug}(\mathrm{ACT}=56.00 \pm 20.40)$ indicated significant reductions $(\mathrm{p}<0.05)$, when compared to the negative control $(\mathrm{NC}=392.00 \pm 14.97$. On the 12 th day of treatment (Figure3), the parasite density levels of groups that received extracts $(\mathrm{G}=64.00 \pm 9.80, \mathrm{~L}=48.00 \pm 14.9, \mathrm{G}$ $\mathrm{L}=56.00 \pm 9.80$ and standard drug (ACT $=24.00 \pm 9.80)$ dropped significantly $(\mathrm{p}<0.05)$ when compared to the negative control $(P C=456.00 \pm 9.80)$. Groups $G$, GL and L showed no significant difference $(p>0.05)$. There was also no significant difference $(\mathrm{p}>0.05)$ between ACT, L and GL groups. Generally, C. limon extract administration induced increased red blood cell lysis in the studied animals.

Table 1 Baseline parasite densities among mice in different treatment groups at day 1

\begin{tabular}{|c|c|}
\hline Treatment groups & Mean parasite densities \\
\hline G & $216.00 \pm 9.80^{a}$ \\
\hline L & $240.00 \pm 17.89^{a}$ \\
\hline ACT & $248.00 \pm 14.97^{a}$ \\
\hline GL & $216.00 \pm 9.79^{a}$ \\
\hline NC & $216.00 \pm 9.80^{a}$ \\
\hline
\end{tabular}

Keys:G = Grape extract; $\mathrm{L}=$ Lemon extract; GL= Grape and Lemon extracts; $\mathrm{ACT}=$ Artemether-Lumenfantrine; $\mathrm{NC}=$ Negative control Results are expressed as means+ standard error of means Mean values with different alphabets as superscript are significantly different $(\mathrm{p}<0.05)$.

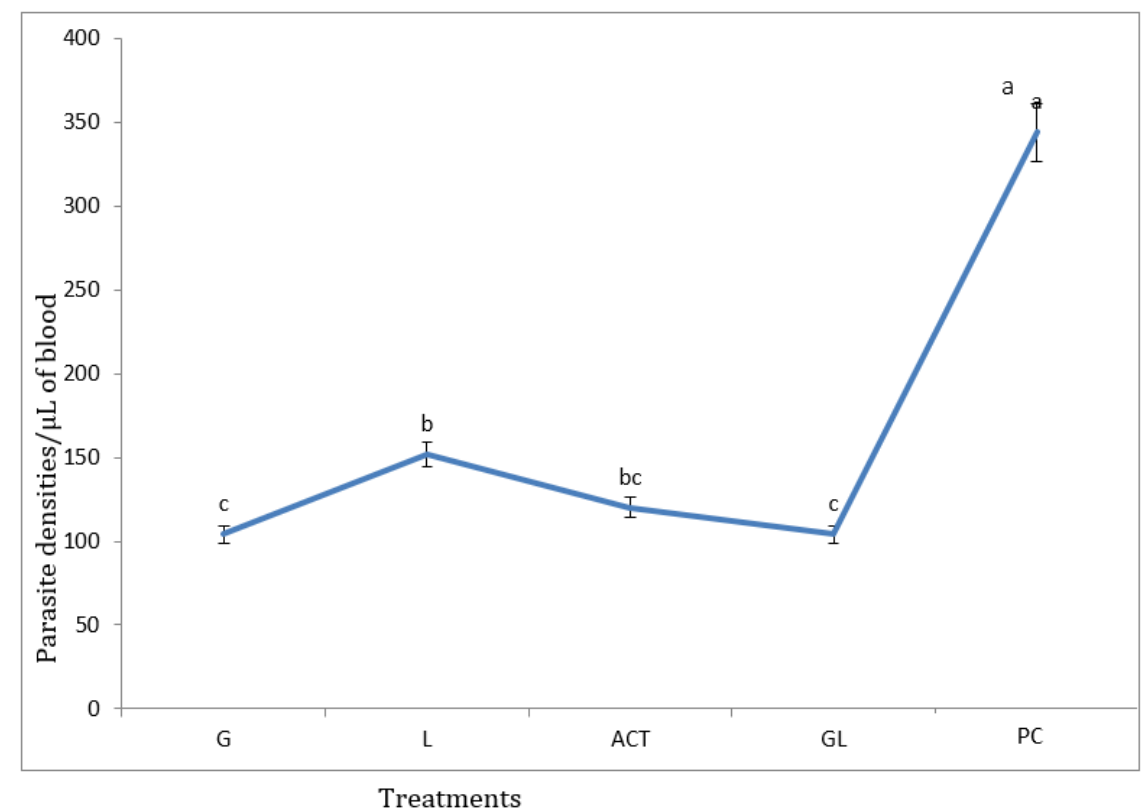

Mean values with different alphabets as superscript are significantly different $(\mathrm{p}<0.05)$

Keys: $\mathrm{G}=$ Grape extract; $\mathrm{L}=$ Lemon extract; $\mathrm{GL}=$ Grape and Lemon extracts; $\mathrm{ACT}=$ Artemether-Lumenfantrine; NC= Negative control.

Figure 1 Parasite densities among mice in different treatment groups at day 6 
World Journal of Biology Pharmacy and Health Sciences, 2021, 08(02), 026-033

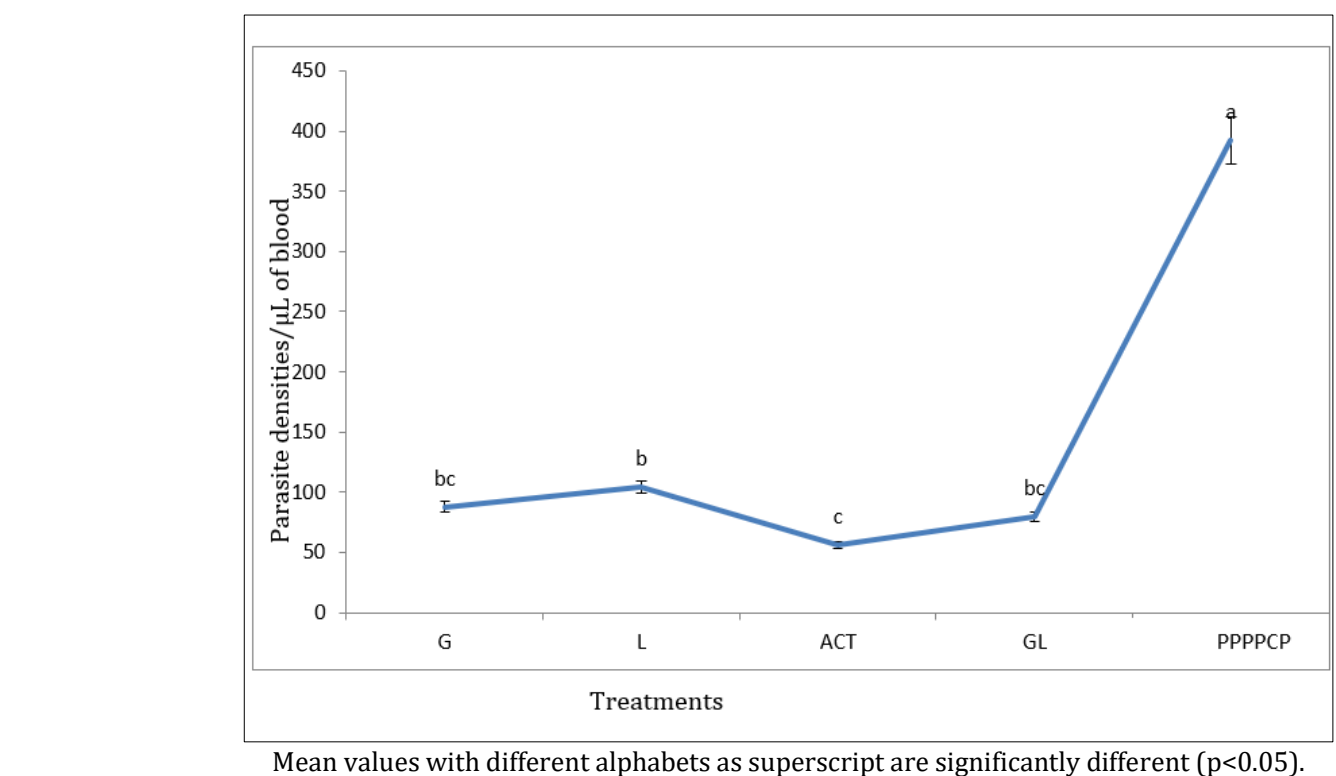

Keys: $\mathrm{G}=$ Grape extract; $\mathrm{L}=$ Lemon extract; $\mathrm{GL}=$ Grape and Lemon extracts; $\mathrm{ACT}=$ Artemether - Lumenfantrine; $\mathrm{NC}=$ Negative control

Figure 2 Parasite densities among mice in different treatment groups at day 9

Table 2 Parasite densities among mice in different treatment groups at day 3

\begin{tabular}{|c|c|}
\hline Treatment groups & Mean parasite densities \\
\hline G & $152.00 \pm 8.00^{\mathrm{b}}$ \\
\hline L & $192.00 \pm 14.97^{\mathrm{b}}$ \\
\hline ACT & $160.00 \pm 17.89^{\mathrm{b}}$ \\
\hline GL & $160.00 \pm 12.65^{\mathrm{b}}$ \\
\hline NC & $312.00 \pm 14.97^{\mathrm{a}}$ \\
\hline
\end{tabular}

Keys:G = Grape extract; L= Lemon extract; GL= Grape and Lemon extracts; ACT= Artemether-Lumenfantrine; NC= Negative control Results are expressed as means+ standard error of means Mean values with different alphabets as superscript are significantly different $(\mathrm{p}<0.05)$.

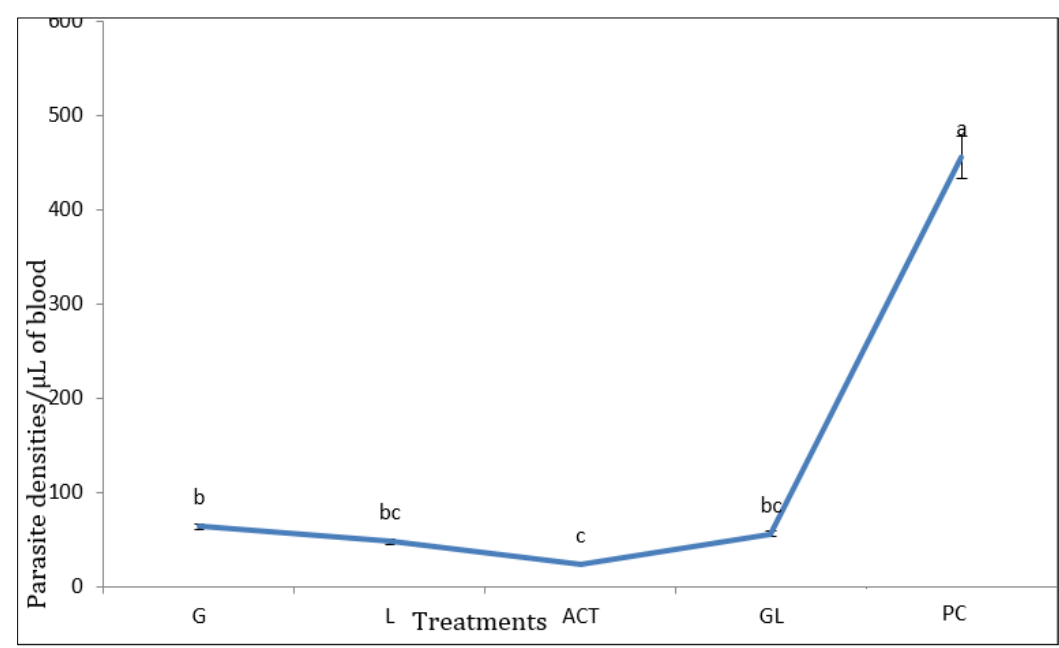

Mean values with different alphabets as superscript are significantly different $(\mathrm{p}<0.05)$.

Keys: G = Grape extract; L= Lemon extract; GL= Grape and Lemon extracts; ACT=Artemether-Lumenfantrine; $\quad$ NC= Positive control.

Figure 3 Parasite densities among mice in different treatment groups at day 12 


\section{Discussion}

In the present study, it was observed that both $C$. paradisi and $C$. limon had antiplasmodial properties both in single and in combined strengths. Reports of several antiplasmodial activities of plant extracts on malaria-infected mice have been documented. Onyegbule et al. [24] reported anti-plasmodial activities of ethanol extracts and fractions of Jatropha gossypifolia in P. berghei- infected mice. Enechi et al. [25] reported a dose- dependent reduction in percentage malaria parasitaemia (increased malaria parasite clearance) among P. berghei-infected mice treated with Fagarazanthoxyloides leaf extracts. Other similar studies on antiplasmodial activities of plant extracts include those of Somsak et al. [26], Zemicheal and Mekonnen [27] and Eluu et al [28]. The antiplasmodial properties of $C$. limon observed in the present study is similar to the findings of Saotoing et al. [16]. The use of $C$. limon extract for treating mice malaria infections in this study indicated increased red blood cell lyses. When the RBCs of another group of mice that were not infected with $P$. berghei but fed with C.limon extract were examined erythrocyte lysis was also observed, though with lesser intensity. This phenomenon of plant extract- induced RBC lysis has been reported by Laser et al. [28]. Lysing of RBCs could be attributed to the mechanism of action of the plant extracts, as has earlier been asserted by Laser et al. [29]. Antimalarial drugs target different stages of the Plasmodium life cycle and could express different stage- specific mechanisms of action during parasite clearance. The schizonticides have been reported to exert their chemotherapeutic effects by inducing premature lyses of parasitized red cells. The presence of phytochemicals in the fruits could be responsible for the phenomenon of observed lyses. Limonin, a phytochemical that is present in $C$. limon has been previously reported to possess some beneficial chemotherapeutic and bioactive effects such as inhibiting retrovirus replication [30], reduction of colon cancer proliferation [31] and anti-obesity effect in mice [32].

The anti-plasmodial effect of C. paradisi extract could also be attributed to the presence of a flavonoid, a phytochemical that contains a compound known as naringin which is normally found in citrus fruits and the substance has been reportedly suspected to be responsible for the bitter taste of grapefruits [33,34]. In fact, the anti-plasmodial activity of many plant extracts has been attributed to either single or combined actions of such metabolites [24,35]. Administration of $C$. paradisi extract to the mice resulted in shrinking of their erythrocytes. Erythrocyte shrinkage could also be attributed to mechanism of action of $C$. paradisi extract during malaria parasite clearance as has been suggested by Ginsburg et al. [36], who observed that the growth of malaria parasites was inhibited when infected erythrocytes were osmotically shrunken in a hypertonic media but the shrinkage was not attributed to parasite dehydration. Based on the observation, Ginsburg suggested that increased viscosity of host cytosol and/or hemoglobin gelatin could be responsible for the shrinking effect, probably through interference with parasite feeding [36].

The use of both $C$. paradisi and $C$. limon in a combined state had great antiplasmodial capacity and still maintained normal RBC morphology, probably due to an ameliorating effect of the flavonoids in both extracts. ACT as a standard drug was used to confirm that the strain of P. berghei ANKA could be susceptible to any other orthodox antimalarial except chloroquine in which it was resistant to. ACT exhibited the highest antiplasmodial activity among all the treatment regimens. This observation is in line with the findings of Somsak et al. [26], who reported highest antimalaria activity of the orthodox medicine in comparison with the plant extracts used in their study. Both $C$. paradisi and $C$. limon have been reported to be rich in vitamin $C$ which functions as an antioxidant for protecting the immune system from free radicals during parasite invasion [19] and based on that important function, [37] advocated the incorporation of vitamin $\mathrm{C}$ in the management of malaria.

\section{Conclusion}

From the present study, it was observed that administration of the extracts of both $C$. paradisi and $C$. limon either in single or in combined strengths, had antiplasmodial properties in the studied animals. It is interesting to note that the strain of the Plasmodium berghei ANKA which has been known to be resistant to chloroquine but susceptible to ACTs was also observed to be susceptible to the two plant extracts used in this study, in both single and combined strengths. ACT had the highest antiplasmodial efficacy whereas. $C$. limon extract showed second highest antiplasmodial effect but exhibited increased RBC lysis. In combination, the extracts (C. paradisi and C. limon) also exhibited antiplasmodial capability that was a little less than that of lemon extract alone, but maintained normal red cell morphology; an advantageous feature that projects it as a better antimalarial in comparison with others. Grapefruit extract showed the lowest level of parasite clearance and also shrinked the red blood cells greatly. In conclusion, effect of the extracts on liver, kidney and gastrointestinal tissues of mice should be investigated before extending their use as malaria drugs to other animals and humans. 


\section{Compliance with ethical standards}

\section{Acknowledgments}

The authors acknowledge and thank the Parasitology Unit of the Faculty of Veterinary Medicine, University of Nigeria, Nsukka and the Laboratory attendants in the Laboratory where the study was carried out.

\section{Disclosure of conflict of interest}

The authors declare that there is no conflict of interest.

\section{Statement of ethical approval}

The study was ethically-approved. All ethical precepts that involve the use of animals for laboratory studies and experiments were dully observed in the study.

\section{References}

[1] Caraballo H. Emergency department management of mosquito-borne illness: malaria, dengue,and West Nile virus. Emergency Medicine Practice. 2014; 16(5): 1-23.

[2] Biamonte MA, Wanner J, Roach KGL. Recent advances in malaria drug discovery. Bioorgarnic and Medical Chemistry. 2013; 23(10): 2829-2843.

[3] Ajayi JB, Agbeyangi AO, Daniel A, Omoboaji I, Magaji HO. Clinical manifestations of Plasmodium berghei ANKA infection in juvenile mice: a short case report. World News of Natural Sciences. 2017; 14: 90-96.

[4] Korenromp E, Williams B, de Vlas S, Gouws E, Gilks C, Ghys E, Nahlen B. Malaria attributable to the HIV-1 epidemic, in sub-Sahara Africa. Emerging Infectious Diseases. 2005; 11(9): 1410-1419.

[5] Adebayo JO, Krettli AU. Potential antimalarials from Nigerian plants: a review. Journal of Ethnopharmacology. 2011; 133: 289-302.

[6] Beare NA, Lewallen S, Taylor TE, Molyneux ME. Redefining cerebral malaria by including malaria retinopathy. Future Microbiology. 2011; 6(3): 349-355.

[7] Roy S, Chetia D, Rudrapal M, Prakash A. Synthesis and antimalarial activity study of some munich bases of 7 chloro-4-aminoquinoline. Medical Chemistry. 2013; 9(3): 379-383.

[8] Rudrapal M, Chetia D, Prakash A. Synthesis, antimalarial and antibacterial ac $\neg$ tivity evaluation of some new 4aminoquonoline derivatives. Medical Chemistry. 2013; 22(8): 3703-3711.

[9] World Health Organization. World Malaria Report. WHO: Geneva Convention. 2015.

[10] World Health Organization. World Malaria Report. Accessed 21 January, 2019.

[11] Center for Disease Control and Prevention. CDC-Global health: Division of Parasitic Diseases and Malaria. 2018.

[12] Hartman TK, Rogerson SJ, Fischer PR. The impact of maternal malaria on newborns. Annals of Tropical Paediatrics. 2010; 30(4): 271-282.

[13] Keeling PJ, Rayner CJ. The origin of malaria: there are more things in heaven and earth. Parasitology. 2014; 142: 16-25.

[14] Bjanquart S, Gascuel O. Mitochondrial genessupport a common origin of rodent malaria parasites and Plasmodium falciparum relatives infecting great apes. BMC Evolutionary Biology. 2011; 11: 70.

[15] Ding XC, Ubben D, Wells TNC. A framework for assessing the risk of resistance for antimalarials in development. Malaria Journal. 11: 292.

[16] Saotoing P, Vroumsia T, Tchuenguem F, Fernand NN, Alexandra M, Messi J. Medicinal plants used in traditional treatment of malaria in Cameroon. Journal of Ecology and the Natural Environment. 2011; 3(3): 104-117.

[17] Molyneux RJ, Lee ST, Gardener DR, Panter KE, James LF. Phytochemicals: the good, the bad and the ugly. Phytochemistry. 2007; 68(22): 2973-2985. 
[18] Burt SA. Essential oils: their antibacterial properties and potential applications in foods: a review. International Journal of Food Microbiology. 2004; 94: 223-253.

[19] Wintergerst ES, Maggini S, Horning DH. Lime enhancing role of vitamin C and zinc and their effects on clinical conditions. Annals of Nutrition and Metabolism. 2006; 50: 85-94.

[20] Carter R, Diggs CI. Plasmodia of rodents. Pages 359-465 in: Parasitic Protozoa, Volume III. Kreier, J.P., Editors. Academic Press, New York, USA.

[21] Kim DH, Jeong KH, Jung UJ, Kim SR. Naring treatment induces neuroprotective effects in a mouse model of Parkinson's disease in vivo, but not enough to restore the lesioned dopaminergic system. Journal of Nutritional Biochemistry. 2016; 28: 140-146.

[22] Cheesebrough M. District Laboratory Practice in Tropical Countries. Part 1. Cambridge University Press, United Kingdom. 2006.

[23] Haggaz AD, El-bashir LM, Adam GK. Estimating malaria parasite density among pregnant women at central Sudan using actual and assumed WBC count. Malaria Journal. 2014; 13: 6.

[24] Onyegbule FA, Bruce SO, Onyekwe ON, Onyealisi OL, Okoye PC. Evaluation of the in vivo antiplasmodial activity of ethanol extract and fractions of Jatropha gossypifolia in Plasmodium berghei -infected mice. Journal of Medicinal Plants Research. 2019; 13(11): 269-279.

[25] Enechi OC, Amah CC, Okagu IU, Ononiwu CP, Azidiegwu VC, Ugwoke EO, Onoh AP, Ndukwe EE. Methanol extracts of Fagarazanthoxyloidesleaves possess antimalarial effects and normalizes haematological and biochemical status of Plasmodium berghei- passage mice. Pharmaceutical Biology. 2019; 57(1): 577-585.

[26] Somsak V, Noilod J, Chachiyo S, Kraithap S. Antimalarial activity of ethanolic leaf extract of Bauhinia strychnifoliainmiceinfected with Plasmodium berghei. Malaria Control and Elimination. 2015.

[27] Zmicheal G, Mekonnen Y. Antiplasmodial activity of Vernoniaadoensisaqeous, methanol and chloroform sensitive strain of Plasmodium bergheiin vivo mice. BMC Research Notes. 2018.

[28] Eluu SC, Oko AO, Osonwa UE, Eze GO, Ngele KK. Evaluation of antiplasmodial activity of ethanol leaf extracts ofLannaeacidonPlasmodiumberghei- infected albino mice. World Journal of Medical Sciences. 2019; 16(3): 134141.

[29] Laser H, Kemp P, Miller N, Lander D, Klein R. Malaria, quinine and red cell lysis. Parasitology. 1975; 71(2): 167181.

[30] Balestrieri E, Pizzimenti F, Ferlazzo A, Giofrè SV, Iannazzo D, Piperno A, Romeo R, Chiacchio MA, Mastino A, MacChi B. Antiviral activity of seed extract from Citrus bergamia towards human retroviruses. Bioorganic and Medicinal Chemistry. 2011; 19(6): 2084-2089.

[31] Chidambara KN, Jayaprakasha GK, Kumar V, Rathore KS, Patil BS. Citrus limoninand its glucoside inhibit colon adenocarcinoma cell proliferation through apoptosis. Journal of Agricultural and Food Chemistry. 2011; 59 (6): 2314-2323.

[32] Ono E, Inoue J, Hashidume T, Shimizu M, Sato R. Anti-obesity and anti-hyperglycemic effects of the dietary citrus limonoidnomilin in mice fed a high-fat diet. Biochemical and Biophysical Research Communications. 2011; 410(3): 677-681.

[33] Yusof S. Naringin contents in local citrus fruits. Food Chemistry. 1990; 37(2): 113-1121.

[34] Renugaderi J, Prabu SM. Naringenin protects against Cadium-induced oxidative renal dysfuntion in rats. Toxicology. 2009; 256: 128-134.

[35] Khaomek P, Ichino C, Ishiyama A, Sekiguchi H, Namatame M, Ruangrunsi N, Saifah E, Kiyohara H, Otogura K, Omura S, Yamada H.In vitro antimalarial activity of prenylated flavonoids from Erythrinafusca. Journal of Natural Medicine. 2008; 62(2):217-220.

[36] Ginsburg H, Handeli S, Friedman S, Gorodestsky R, Krugliak M. Effects of red blood cell potassium and hypertonicity on the growth of Plasmodium falciparum in culture. Zeitschriftfurparasitenkunde. 1986; 72: 85199.

[37] Marve E, Her-El R, Golenser J, Cohen A, Kitrossky N, Chevion M. The effects of ascorbate-induced free radicals on Plasmodium falciparum. Journal of Tropical Medicine and Parasitology. 1992; 43:17-23. 